\title{
Brentano et le réalisme moral
}

Par GEMMO IOCCO

Università degli Studi di Parma

Résumé Dans ses écrits sur l'éthique, Brentano affirme que les propriétés morales - comme «bon » - sont l'objet d'une représentation intuitive non sensible dont le contenu est de nature «mentale ». Si la nature mentale de ces contenus en garantit le caractère d'évidence, elle en complexifie aussi l'élucidation ontologique et épistémologique, qui doit tenir compte de la classification brentanienne des actes psychiques. C'est justement cette complexité constitutive qui a fait de la théorie éthique de Brentano un passage obligé pour beaucoup de courants philosophiques importants du $\mathrm{XX}^{\mathrm{e}}$ siècle, par exemple pour la phénoménologie des valeurs et l'intuitionnisme éthique. Le but de cette étude est de rappeler les aspects les plus significatifs de la théorie éthique de Brentano et de montrer si — et si oui, en quel sens elle peut être qualifiée de « réaliste».

Que veut dire être un réaliste moral ? Le réaliste moral affirme généralement qu'il existe une réalité moralement connotée indépendamment de l'esprit, qu'on essaie de représenter lorsqu'on formule des jugements relatifs à ce qui est juste ou injuste. Toutefois, ce qui fait débat au sein même du réalisme, c'est la nature même de cette réalité. On peut également définir comme réaliste, dans un sens très spécifique, la théorie de la valeur proposée par Franz Brentano dans la phase initiale de sa réflexion philosophique et psychologique (Baumgartner \& Pasquarella 2004 ; Kriegel 2018 : 187-282) : cela démontre la plasticité du concept de « réalisme », qui peut désigner une position - comme celle de Brentano - axée sur l'idée que la psychologie, au sens de l'étude des activités psychiques, est la seule méthode qu'on peut légitimement qualifier d' « évidente ». 
Selon Brentano, on peut répartir la multiplicité des activités psychiques en trois catégories ou classes différentes, à savoir : les présentations (Vorstellungen), les jugements (Urteile) et les mouvements affectifs (Gemütsbewegungen). Ce qui différencie ces classes n'est pas l'objet, mais la modalité sous laquelle advient la référence à l'objet. Si je pense à Dieu, j'ai une présentation ; si j'affirme «Dieu existe» je formule un jugement; si je pense «j'espère que Dieu me protégera », j'exprime un désir, c'est-à-dire un mouvement affectif. L'analyse des phénomènes de la sphère affective, en référence aux relations de dépendance entre les phénomènes des trois classes, a le mérite de faire ressortir les spécificités du modèle gnoséologique proposé par Brentano. En effet, l'expérience nous met en présence de phénomènes structurés et complexes qui sont imprégnés de valeur : comme Oskar Kraus le remarque dans l'Introduction à l'édition de 1925 de la Psychologie du point de vue empirique, « les perceptions sensibles de plaisir ou de déplaisir sont le début de notre vie consciente », tandis que les présentations «n'apparaissent avec évidence qu'à travers une activité conceptuelle supérieure » (Kraus 1972 [1925] : XIX). L'observation de Kraus - qui revendique la primauté de la dimension émotionnelle (Kraus 1937) — risque de nous fourvoyer si l'on ne tient pas compte de la différence entre le point de vue descriptif et le point de vue génétique. Car les émotions, selon Brentano, sont nécessairement fondées sur des présentations. Il s'agit pour Brentano, d'une part, de reconnaître aux émotions un statut intentionnel spécifique et, d'autre part, de démontrer que les phénomènes de la troisième classe sont nécessairement fondés sur des présentations. Afin de légitimer la préséance des présentations par rapport aux phénomènes des deuxième et troisième classes, Brentano affirme que la composante affective n'est pas ressentie directement, car les présentations sont des modalités de référence intentionnelle affectivement neutres. Il s'agit également de déterminer précisément le rapport entre présentation et sentiment pour comprendre s'il est vraiment possible que des sentiments non fondés sur la présentation existent. Dans l'optique de Brentano, cependant, revendiquer pour certaines classes de mouvements affectifs un statut différent par rapport au statut intentionnel serait une contradiction gnoséologique :

Pour certaines sensations de plaisir ou de douleur, nous admettons bien qu'on puisse nier l'existence d'une présentation au sens où nous l'entendons. On est, en tout cas, tenté de le faire. Cela s'applique aux sentiments provoqués par une coupure ou une brûlure. Si vous vous coupez, vous n'éprouvez d'ordinaire aucune perception de contact ; si vous vous brûlez, aucune perception de chaleur; dans les deux cas, il ne semble y avoir que de la douleur. Il est hors 
de doute, néanmoins, que dans ces cas également le sentiment repose sur une présentation. Nous avons toujours la présentation d'une localisation précise que nous lions d'ordinaire à telle ou telle partie visible et palpable de notre corps (Brentano 1973 [1874]/2008 : 116/95-96).

En effet, du point de vue épistémique, ces sensations de plaisir ou de douleur sont des représentations ou manifestations fondées sur des représentations. ${ }^{1}$. Du point de vue génétique, au contraire, le fait qu'une représentation puisse naître de la sensation ou de la fantaisie est secondaire par rapport à la priorité gnoséologique qu'elle possède par rapport à d'autres manifestations psychiques. Le terme «représentation » se réfère donc tant aux représentations intuitives qui proviennent de la perception qu'aux représentations conceptuelles: dans le premier cas on assume l'objet comme s'il était directement perçu ; dans l'autre au contraire, l'objet qui se manifeste à l'esprit est pensé à travers les concepts sur lesquels il est fondé, et il s'agit donc, dans ce cas, d'un objet purement mental.

\section{Les émotions et la psychologie de l'évaluation}

À l'intérieur de la troisième classe des activités psychiques, on trouve aussi bien les actes du sentiment que les actes de la volonté; Brentano utilise l'expression Gemütsbewegungen pour définir une classe de phénomènes qui rassemble les phénomènes d'amour et de haine, d'inclination, de répulsion, de joie et de douleur, unissant ainsi le sentiment et la volonté ${ }^{2}$. Pour essayer de définir les analogies et les différences entre les phénomènes de la troisième classe et ceux de la deuxième classe, il est utile d'observer qu'ils partagent ein Gegensatz der intentionalen Beziehung : "L'opposition affective entre l'amour et la haine correspond, comme je l'ai montré, à l'opposi-

\footnotetext{
${ }^{1}$ Afin d'éviter toute équivoque, nous traduisons toujours Vorstellung au sens brentanien par «présentation», et employons « représentation» pour d'autres types de phénomènes psychiques.

${ }^{2}$ Toujours en référence aux distinctions internes à la troisième classe de phénomènes psychiques, la dispute avec Stumpf à propos de la distinction entre émotions et Gefühlsempfindungen est particulièrement intéressante. Stumpf, en effet, affirme qu'il existe une différence spécifique entre les Gefühlsempfindungen, qui sont des qualités sensibles comme le son et la couleur, et les émotions définies en tant qu'états émotionnels dirigés vers des objets ( $c f$. Stumpf 1906). Pour comprendre les analogies et les différences entre les deux connotations, voir (Fisette 2011). Sur l'approche de Brentano, voir (Massin 2013).
} 
tion, sur le plan du jugement, entre l'acceptation et le rejet» (Brentano 1973/2008: 152/298). Il existe donc une analogie formelle significative (Seron 2008) entre les phénomènes rentrant dans la classe du jugement et ceux rentrant dans la classe des mouvements affectifs ou émotions : de la même façon qu'un jugement doit nécessairement être positif ou négatif, les émotions apparaissent comme des relations intentionnelles d'amour ou de haine envers un objet déterminé. Si l'on empruntait la terminologie de Husserl, on pourrait dire que jugements et sentiments possèdent un « caractère d'acte » que les représentations, au contraire, ne possèdent pas. Jugements et sentiments sont fondés sur les présentations mais ne se réduisent pas à celles-ci — bien entendu, les mouvements affectifs sont fondés sur les présentations de façon indirecte, étant donné que leur référence spécifique est le jugement. Du point de vue ontologique et qualitatif, les présentations sont neutres tandis que les jugements et les mouvements affectifs — dans leurs formes multiples ${ }^{1}$ — sont nécessairement positifs ou négatifs. Les présentations de la valeur ou de la non-valeur dérivent ainsi de l'expérience interne de ces phénomènes. S'il s'agit donc, dans le cas du jugement, d'affirmer ou de nier quelque chose, dans celui des émotions on se trouve en face de phénomènes d'amour ou de haine qui se sont révélés justes. Le fait de percevoir qu'une action déterminée est juste ou ne l'est pas est une forme de référence intentionnelle sui generis qui nécessite cependant d'être fondée sur le jugement et donc sur la présentation. Brentano, qui reprend la terminologie de Leibniz, appelle ces formes d'activité psychique vérités de raison ou axiomes, car elles possèdent une valeur épistémologique garantie par la perception interne. L'affirmation de Brentano concernant le concept de bien rapporté aux émotions est éloquente en ce sens :

Cette sphère (la sphère des émotions) renferme une variété encore plus grande d'activités par rapport à celle du jugement. Cette dernière renferme des distinctions en référence à l'objet, et en particulier, entre jugement sensible et intellectuel ; des distinctions selon la qualité du jugement (reconnaissance ou refus) ; la distinction entre jugement thétique et jugement prédicatif, entre jugement «aveugle » et jugement évident et entre jugement motivé et jugement immotivé ; les distinctions de modalité, assertorique ou apodictique ; et

\footnotetext{
${ }^{1}$ Le fait qu'elles soient «multiples » semblerait conduire à la nécessité d'une articulation plus détaillée des activités psychiques de type judicatif et émotionnel. On a, en effet, l'impression qu'à travers l'analyse graduelle des différentes formes d'activités psychiques, Brentano a maintenu la tripartition présentation - jugements-mouvements affectifs dans le seul but de ne pas renier l'une des thèses formelles fondamentales de son analyse précédente.
} 
enfin les distinctions temporelles, par rapport au fait que ce qui est jugé est présent, passé ou futur. À l'intérieur de la sphère des émotions on trouve des distinctions correspondantes. Il existe ici aussi des différences par rapport à l'objet et plus exactement entre objet sensible et objet intellectuel ; distinctions de qualité - amour/haine ; et la distinction entre ce qui est « aveugle » et ce qui est caractérisé comme correct ainsi qu'entre ce qui est motivé et ce qui ne l'est pas. Mais à côté de ces différences il existe aussi d'autres distinctions par rapport à la position dans laquelle la personne qui « aime » croit se trouver en face de la chose qu'elle aime. Il y a des distinctions entre joie, douleur, désir, espoir et volonté (Brentano 1952 : 185 ; je traduis).

Les phénomènes de la troisième classe ne possèdent pas de caractère normatif établi, mais ce sont de simples actes d'amour et de haine qui se manifestent de manière objective. C'est-à-dire que les actes de préférence sur lesquels se fondent les choix renvoient à la dimension subjective, car ils ne répondent pas entièrement à des lois formelles et objectives. Pour éviter le risque d'une éthique « subjectiviste » envers l'objet de représentation, Brentano distingue deux modalités différentes de plaisir et de déplaisir ( $c f$. Albertazzi 2006 : 298-299) : a) un type de plaisir étroitement lié à l'habitude et b) un type de plaisir lié aux actes de désirer quelque chose de bon ou de mauvais. C'est surtout le second type de plaisir qui se révèle important du point de vue éthique et normatif.

Pour résumer les aspects les plus significatifs du cognitivisme moral proposé par Brentano, on peut affirmer que : a) tout phénomène de la troisième classe possède un objet intentionnel déterminé et présuppose un jugement ; b) ces phénomènes ne sont cependant pas des objets « ordinaires » mais des propriétés axiologiques spécifiques ou valeurs; c) si les phénomènes de la troisième classe se distinguent des présentations et des jugements en relation avec la modalité de référence intentionnelle, ils possèdent, du point de vue gnoséologique, une valence intrinsèquement cognitive et leur influence axiologique réside également en cela. Enfin, du point de vue cognitif, d) les Gemütsbewegungen expriment une certaine forme de préférence.

\section{La théorie de la valeur entre réalisme et psychologie}

Le texte de la conférence rédigé pour la Wiener juristische Gesellschaft en 1889 et intitulé L'origine de la connaissance morale constitue, selon 
l'opinion majoritaire chez les spécialistes, la synthèse la plus achevée de la réflexion éthique de Brentano' ${ }^{1}$.

Comme l'indique le titre même de la conférence, il s'agit de trouver l'origine du concept de bien et de l'intégrer à la classification des actes psychiques en le différenciant des phénomènes des deux autres classes. Brentano affirme que le concept de «bon » dérive de la perception interne et il en propose une définition discursive (McAlister 1982: 83) fondée sur l'équivalence conceptuelle parfaite entre ce qui est bon et ce qui est digne d'être aimé. Les phénomènes en question sont des types spécifiques d'émotions, qui rendent possible le repérage d'axiomes a priori des valeurs et de la préférence. Brentano précise qu'attribuer à une certaine connaissance le statut d'a priori, ne signifie pas que les concepts dont il s'agit sont donnés sans perception ou aperception, car «ce qui distingue le type de connaissance dont il est ici question des autres, est le fait que l'on doit percevoir ou apercevoir certains actes d'amour et pas seulement certaines connaissances intellectuelles » (Brentano 1969: 111). Les processus d'évaluation qui rendent possible la connaissance de ce qui est juste et de ce qui est injuste se configurent comme des modalités intentionnelles qui exigent une composante «qualitative et psychologique » ultérieure par rapport aux activités psychiques reconductibles aux classes de la présentation et du jugement. Les actes d'évaluation et de préférence qui sont reconnus comme justes fournissent par conséquent les normes pratiques qui indiquent quelles fins doivent être poursuivies par les sujets moraux. Il faut cependant distinguer les concepts de valeur et de bonté de ceux de fin pratique et de bien pratique, car les premiers peuvent varier d'une personne à l'autre tandis que les

\footnotetext{
${ }^{1}$ Ce texte (Brentano 1969) a été traduit en anglais par Cecil Hague dès 1902, sous le titre The Origin of our Knowledge of Right and Wrong. Brentano y tente de tracer de façon spécifique une théorie de la valeur. Il est toutefois impossible de comprendre les termes dans lesquels le problème éthique et moral est articulé par Brentano sans avoir placé ces réflexions à l'intérieur du problème de la classification des activités psychiques ébauchée dans le premier volume de la Psychologie (1874) et développée dans les appendices à la réédition partielle du second volume (1911), à travers l'approfondissement de la tripartition des activités psychiques en présentations, jugements et phénomènes émotifs. Il faut ajouter à la conférence de 1889 les cours de 1876-1894 publiés en 1952 par F. Mayer-Hillebrand sous le titre Grundlegung und Aufbau der Ethik. Il existe également différents essais qui traitent de façon plus ou moins spécifique des problématiques d'ordre moral, par exemple Das Recht auf den Selbst (1893), Über der apriorischen Charakter der ethischen Prinzipien (1904), Lieben und Hassen (1907), Über Gemütsentscheidungen und die Formulierung des obersten Sittengesetzes (1908) (cf. Albertazzi 2006 : 295-296).
} 
seconds doivent valoir pour tous. Les valeurs ne doivent pas être considérées comme des entités indépendantes dans le monde objectif, car elles ont besoin pour se constituer de la présence d'un sujet.

Selon Brentano, on dit qu'une chose est bonne quand l'appréciation qui accompagne la représentation de l'objet en question est juste : être bon est conceptuellement équivalent à être digne d'être apprécié et donc aimé. Le bien ne coïncide évidemment pas avec l'utile et encore moins avec l'agréable ; il s'ensuit que le concept de bien que propose Brentano est une valeur intrinsèque, une valeur primaire et non pas instrumentale. Ce modèle éthique se construit à travers des formes de connaissance qui sont, en même temps, déduites a priori de l'expérience puisqu'elles ne se constituent pas à la suite d'un processus de nature synthétique. La justesse d'un acte d'amour, toutefois, n'est pas toujours saisissable :

Nous ne pouvons, en effet, nous dissimuler ceci que nous n'avons aucune garantie du fait que tout ce qui est bon provoque en nous un amour considéré comme juste. Dès que ce n'est plus le cas, notre critère n'est plus valable, et le bien fait tout simplement défaut, autant à notre connaissance qu'à notre réflexion pratique » (Brentano 1969/2003 : 24/67).

Il s'ensuit que l'objectif de Brentano, quand il applique la méthode psychologique et descriptive à la sphère éthique ${ }^{1}$, est de déterminer les principes qui règlent nos connaissances éthiques à partir d'une perspective d'analyse complètement nouvelle, basée sur la thèse selon laquelle les sentiments revêtent un rôle d'importance primordiale dans le processus cognitif. Cependant, le renvoi au sentiment n'est pas légitimé de façon subjective, mais objective. Les questions préliminaires auxquelles on cherche à donner une réponse sont ainsi les suivantes :

Y a-t-il une loi morale naturelle au sens où, immuable et universellement valable en elle-même, elle vaudrait pour les hommes en tout lieu et de tout temps, c'est-à-dire pour tous les êtres doués de pensée et de sentiment? » (Brentano 1969/2003: 9/40).

La réponse de Brentano à ces deux interrogations est affirmative en vertu d'une certaine analogie formelle qui existe entre les lois de la logique et les lois qui règlent les actes de la volonté :

\footnotetext{
${ }^{1}$ En référence aux prémisses développées par Brentano dans sa réflexion sur les conditions de possibilité de la conscience morale ( $c f$. McAlister 1982 : 43).
}

7

Bull. anal. phén. XIV 3 (2018)

https://popups.uliege.be/1782-2041/ ( 2018 ULiège BAP 
Les nécessités logiques sont des règles, naturellement valables, auxquelles se soumet le jugement ; c'est-à-dire qu'il est nécessaire de les observer, car le jugement conforme à ces règles est sûr, tandis que celui qui s'en écarte est exposé à l'erreur; il s'agit donc d'un avantage naturel que possède la démarche réflexive réglée par rapport à celle qui procéderait en dépit de toute règle. Or, dans le domaine moral, c'est également d'un tel privilège naturel qu'il doit s'agir, ainsi que d'une règle qui s'y fonderait, et non pas du commandement issu d'une volonté étrangère (Brentano 1969/2003 : 12/43).

La supériorité intérieure (innerer Vorzug) est ce qui «distingue la volonté morale de la volonté immorale, qui différencie le jugement et la déduction vrais et pertinents des préjugés et des paralogismes » (Brentano 1969/2003 : $13 / 44)$.

La perception de la justesse d'une action ne dépend pas exclusivement de la perception de certaines caractéristiques vérifiables intuitivement, mais également de la disposition du sujet. À l'opposé de ce que Max Scheler affirme, d'après Brentano, les qualités de valeur ne sont pas matérielles, mais mentales, et ce fait en garantit le caractère évident. S'il est certes possible d'être victime d'une illusion et de confondre un mannequin avec une personne réelle, il n'est pas possible de se tromper sur la justesse de l'intention d'aider un ami en difficulté. La perception intérieure, pour utiliser une expression de Scheler, n'est pas sujette à auto-illusion. Une autre différence entre les théories de la valeur de Brentano et de Scheler est illustrée par les termes que les deux penseurs utilisent pour caractériser la préférence. Pour Brentano, la fiabilité épistémologique de la préférence repose sur le fait qu'elle est perçue intérieurement. Pour Scheler en revanche, les valeurs ne fusent (blitzen) pas tant dans la perception ou dans l'observation interne « que dans le rapport vivant senti, avec le monde, dans le fait de préférer (Vorziehen) et de postposer (Nachsetzen), dans le fait d'aimer et de haïr, c'est-à-dire dans l'accomplissement de telles fonctions et actes intentionnels » (Scheler $2000:$ 87). Scheler définit le fait de préférer comme un acte spécifique de la connaissance axiologique (Werterkenntnis) qui permet de saisir la supériorité d'une valeur par rapport à une autre. Ce que Brentano appelle «préférence » ressemble cependant davantage à cette forme de préférence que Scheler définit comme a priori et qui «devient évidente dans le fait même de préférer» (Scheler 2000 : 105). Toutefois, le concept de préférabilité intrinsèque (innere Vorzüglichkeit) est fragile du point de vue théorique, car il est le résultat d'une généralisation. Du point de vue constructif, l'identification psychologique d'une norme morale spécifique semble résulter de «ce principe non négligeable que le domaine 
du bien pratique suprême n'est autre que l'ensemble de la sphère soumise à l'influence de notre raison, pour autant qu'il soit possible d'y réaliser un bien » (Brentano 1969/2003 : 30/75).

Le modèle éthique objectif proposé par Brentano repose donc sur la constatation que la supériorité de l'action moralement juste ne provient pas d'une apparence esthétique (ästhetischer Erscheinung), mais qu'elle est « intrinsèque », c'est-à-dire qu'elle possède une justesse interne (innere Richtigkeit) qui lui est propre (Danielsson et Olson 2007). De même, les attributs moraux et immoraux se réfèrent, avec des nuances différentes, à la volonté : dans de nombreux cas, on désire une chose parce qu'on la considère comme un moyen pour satisfaire un vouloir ultérieur - naturellement, ce qui est poursuivi comme moyen pour atteindre une fin déterminée est une forme de vouloir relative et instrumentale, et donc non intrinsèque.

Le « motif éthique » authentique (das ethische Motiv) réside donc dans la croyance qu'il existe une supériorité intrinsèque considérée comme la fin vers laquelle une volonté moralement juste doit tendre. D'après Brentano, la voie entreprise au dix-huitième siècle - l'idée que les êtres humains tendent naturellement vers les mêmes fins, à savoir vers le plaisir personnel — s'est révélée fallacieuse parce qu'elle n'a pas su saisir l'objectif principal d'une théorie éthique correcte, soit l'identification d'un principe intrinsèquement valable et évident. La seule réponse adéquate à la question de savoir quelles fins sont dignes d'être poursuivies est de choisir le meilleur (Brentano 1969/2003 : 15/47). Brentano affirme toutefois que même cette détermination n'est pas définitive et fondatrice, car elle n'explique pas en quoi une option est meilleure qu'une autre et elle conduit à se demander ce qui peut être défini comme «bon » (Kriegel 2017).

La notion de «bien » trouve son origine dans des représentations intuitives (in anschaulichen Vorstellungen) dont le contenu est cependant psychologique (Brentano 1969/2003 : 16/48) ; la notion de bien «ainsi que celle du vrai, qu'à juste titre on lui juxtapose en la considérant comme apparentée, sont empruntées au domaine des représentations dont le contenu est d'ordre psychique »(Brentano 1969/2003 : 16/48). Les représentations intuitives possédant un contenu physique se différencient selon de multiples critères, spécialement en fonction du contenu psychique lui-même :

Les mouvements affectifs d'ordre sensible constituent ce qu'on appelle des émotions (Affekte). Parmi ces émotions il faut ranger déjà le plaisir et la douleur où l'on a vu souvent des qualités sensibles plutôt que des mouvements affectifs, imaginant qu'on éprouve le plaisir et la douleur comme on voit les couleurs et comme on entend les sons. Pour se rendre compte que 
c'est là une erreur, il suffit de remarquer qu'on peut douter de l'existence du bleu ou du rouge, non de celle du plaisir ou de la douleur. Il ne s'agit pas là de pures qualités sensibles, ni d'ailleurs de relations psychiques qui auraient comme objets directs des qualités sensibles. Leur objet est psychique, c'est la sensation même de certaines qualités sensibles qui est agréable ou désagréable (Brentano 1874/2008: 80/443).

Le concept de bien possède un caractère épistémologique bien particulier. En effet, si d'un côté on ne peut mettre en question le fait que la représentation d'objets noétiques — par exemple l'égalité de la somme des trois angles d'un triangle à deux droits - ne s'accompagne pas nécessairement d'une intuition d'un objet sensible - ce triangle déterminé —, de l'autre, il n'est pas possible d'objectiver le concept de bien à travers la représentation noétique, bien qu'il possède une validité a priori. Le concept de bien trouve son origine dans une représentation intuitive où ce qui est représenté est un objet psychique, perçu intérieurement et doté de validité a priori. Il faut donc comprendre de quelle façon un objet, une attitude ou une conduite peuvent être évalués par le biais d'une opération de type comparatif : sa supériorité consiste dans le fait d'être «préféré ».

En référence à ce que Husserl définirait comme la sphère axiologique formelle, la règle fondamentale est le critère de préférabilité : quand on dit qu'une chose bonne est meilleure qu'une autre, on entend dire qu'on la préfère à une autre. Le caractère apodictique de ce critère étant une activité psychique, il est garanti par la perception interne et il est obtenu à travers un processus intentionnel de référence de second niveau, analogue à celui qui permet l'individuation du concept de « vrai ».

\section{Brentano, Moore et le réalisme moral}

Si l'on argumente en faveur de la possibilité d'une représentation intuitive de ce qui est bon, la position éthique de Brentano semblerait en partie assimilable à une position de type intuitionniste. L'intuitionnisme éthique joue un rôle central dans le débat contemporain ${ }^{1}$. Présupposant l'existence de termes moraux «primitifs» - considérés comme relations, qualités ou concepts non naturels —, l'intuitionnisme éthique affirme qu'ils sont par nature indéfinissables. En vertu de cette tension théorique, l'intuitionnisme doit être

\footnotetext{
${ }^{1}$ Pour une mise en contexte plus précise de l'intuitionnisme éthique à l'intérieur de l'horizon méta-éthique contemporain, voir le volume (Huemer 2005). Pour plus de détails sur l'intuitionnisme éthique, voir (Stratton-Lake 2006).
} 
rangé parmi les théories méta-éthiques de matrice réaliste. En effet, si les termes éthiques originaires sont donnés comme indéfinissables, la réalité qu'ils représentent est une réalité objective qui peut et doit être connue directement.

Il s'ensuit que les propriétés morales sont considérées comme objectives et irréductibles : le «bon », par exemple, se réfère aux propriétés que les choses possèdent de manière tout à fait indépendante de nos attitudes envers elles. La justesse et la bonté sont considérées sous l'angle des caractéristiques objectives présentes dans le monde: un jugement est reconnu comme «bon » et «correct» en vertu du fait qu'il possède une certaine propriété de valeur, c'est-à-dire la propriété d'être bon. Ainsi, la fin ultime d'une position philosophique d'ordre méta-éthique et réaliste comme l'intuitionnisme est d'expliquer la nature des valeurs, la manière dont elles se constituent et la méthodologie qui nous permet de les saisir. L'identification et la reconnaissance d'une valeur déterminée soulève cependant des problèmes ontologiques, gnoséologiques et épistémologiques. Dans le cas précis de l'individualisme éthique, on appelle «intuitions éthiques» les intuitions dont le contenu est une proposition judicative; il s'ensuit que les «expériences liées aux valeurs » ne sont que des phénomènes mentaux qui appartiennent à la catégorie des émotions : elles peuvent être « positives » appréciation, admiration, désir — ou «négatives» — désapprobation, dégoût, mépris. La vérité des croyances qui s'expriment par des jugements moraux dépend donc de la présence ou de l'absence des propriétés relatives aux objets de ces propositions.

La position philosophique intuitionniste se révélerait aporétique si elle ne prêtait pas une attention particulière à la problématique de la valeur, de sa constitution et des modalités par lesquelles il est possible de la reconnaître et de la saisir. La détermination spécifique de la valeur d'un jugement particulier, d'un désir ou d'un vouloir relève de l'axiologie. La pensée intuitionniste essaie donc de trouver un point de rencontre entre l'exigence d'une méthodologie scientifique et évidente - que certains appellent axiologie formelle - et son incontournable fondation empirique et matérielle, définie canoniquement comme la relation faits-valeurs. De manière plus circonscrite, l'axiologie devrait permettre de déterminer les conditions de possibilité et de légitimité de la valeur dite intrinsèque ${ }^{1}$.

Si d'un côté cette conception de l'éthique comme recherche de l'origine d'une Erkenntnis von Gut und Böse a eu le mérite évident de fournir

\footnotetext{
${ }^{1}$ Sur le concept de «valeur intrinsèque », voir le volume (Rønnow-Rasmussen \& Zimmermann 2005). En référence à l'intuitionnisme éthique, voir (Audi 2004).
}

11 
un substrat conceptuel pour une éthique axiologique, de l'autre son renvoi à la justesse interne des actes d'amour et de préférence se révèle épistémologiquement indécis et finalement assez obscur ${ }^{1}$. Cette ambiguïté constitutive explique que l'étiquette de "réalisme immanent» que l'on applique à la théorie de la connaissance de Brentano ne vaut que partiellement pour sa réflexion éthique. Le réalisme immanent qui caractérise la théorie de la connaissance de Brentano se transforme, dans la sphère éthique, en une sorte d'objectivisme émotionnel fondé sur la thèse que ce qui est juste est reconnu comme tel, grâce à une modalité de référence intentionnelle de type émotionnel, qui est à son tour reconnue par la perception interne comme juste. Bien que Brentano identifie un acte spécifique à travers lequel le sujet est capable d'apprendre les concepts moraux, par exemple le bien, le fait que le contenu d'un tel acte soit mental et qu'il réclame une forme spécifique d'auto-perception - la perception de la justesse — ne doit pas induire à penser qu'il existe différents types de réalité (Montague 2017). Par objectivisme émotionnel, on entend l'idée que les émotions sont des évaluations qui se focalisent sur des aspects qualitatifs différents par rapport aux jugements.

Pour analyser ce concept, prenons l'exemple d'une émotion qui est normalement considérée comme juste : l'amour pour le savoir. À un niveau général, on tend en effet à considérer que la connaissance est préférable à l'ignorance. Selon le modèle éthique élaboré par Brentano, cette évidence est fondée sur une représentation qui, à son tour, se base sur la reconnaissance de la préférabilité axiologique du savoir par rapport au non-savoir. Cette représentation, qui est une forme de référence intentionnelle, se configure à la fois comme une forme de jugement évident - le jugement universel selon lequel la connaissance est un bien en soi - et comme un sentiment émotionnel «correctement caractérisé » - le sentiment d'amour pour la connaissance et le sentiment de haine pour l'ignorance. De cette façon, Brentano montre comment notre connaissance morale doit avoir son origine dans une représentation intuitive. Du point de vue épistémologique, on doit cependant souligner que Brentano affirme à la fois que les valeurs sont «senties » et que cette forme spécifique de référence intentionnelle est " greffée » sur l'activité psychique judicative. Les valeurs ne sont reconnues comme telles que si elles sont perçues comme justes.

${ }^{1}$ Cette « obscurité » consiste selon Findlay dans le fait que si, d'un côté, Brentano affirme que certains actes sont caractérisés par une certaine justesse interne, de l'autre, il n'explique pas comment cette justesse interne doit être interprétée ( $c f$. Findlay $1970: 21)$. 
Dans la mesure où elle affirme la possibilité d'une représentation intuitive de ce qui est bon, la théorie éthique de Brentano semblerait en partie assimilable à une position du genre «intuitionniste»(Olson $2017 \mathrm{a}-\mathrm{b})$. On peut en déduire que les assertions fondamentales de l'intuitionnisme se révèlent formellement compatibles avec celles qui sont à la base de la réflexion éthique de Brentano. G.E. Moore lui-même se rendait compte de cette compatibilité essentielle quand il reconnaissait dans Vom Ursprung sittilicher Erkenntnis une exposition radicalement nouvelle des principes fondamentaux de l'éthique (Moore 1903 : 115) ${ }^{1}$. Le plus grand mérite des réflexions de Brentano serait, selon Moore, le fait de reconnaître que « toutes les vérités de la forme "x est bon en soi" sont logiquement indépendantes de toute vérité sur ce qui existe. Aucune proposition éthique de ce genre n'est

${ }^{1}$ L'intérêt (historiquement incontestable) de Moore pour l'éthique de Brentano a sans aucun doute influencé de façon décisive la naissance d'une axiological ethics. Oskar Kraus avait déjà souligné un tel rapprochement en 1937, dans son analyse historique et critique des différentes formes de Werttheorien (cf. Kraus 1937 : 205 214). Un pas ultérieur dans cette direction a été accompli par Findlay qui, dans Axiological Ethics, cernait principalement trois orientations parallèles: a) les positions psychologiques et objectives de Brentano et de Meinong ; b) les positions intuitionnistes de Moore, Rashdall et Ross ; c) les positions «phénoménologiques » de Scheler et de Hartmann. Si ces dernières servent généralement de coordonnées historiques générales d'un point de vue conceptuel, celui qui a construit un parallélisme spécifique entre Brentano et Moore est Roderick Chisholm, en focalisant son attention sur le concept de valeur intrinsèque ( $c f$. Chisholm 1986). En référence au concept de «valeur intrinsèque » élaboré par Brentano et par Moore, Thomas Baldwin remarque justement que Moore et Brentano se trouvent en profond désaccord sur ce que l'on pourrait définir une «métaphysique de la valeur intrinsèque ». Moore adopte une position abstraite et réaliste alors que Brentano interprète la valeur intrinsèque en se référant au concept de digne d'être aimé. Si, d'un côté, la position de Brentano présente de nombreux avantages par rapport à celle de Moore, de l'autre elle conduit inévitablement à se demander par quelle opération épistémologique particulière il est possible d'établir qu'une chose est digne d'être aimée. Baldwin suggère de répondre à cette question sur la base d'une «défense du sens commun » et de la survenance relative du moral sur le naturel, théorisée par Moore. Toutefois, cette position elle-même est insuffisante si l'on ne tient pas compte des sentiments naturels. En outre, elle conduit à une sorte de naturalisme éthique explicitement rejeté par Brentano ( $c f$. Baldwin 1999). Tels sont certains des aspects les plus problématiques des tentatives visant à trouver un point de rencontre entre les deux positions. En ce qui concerne l'aspect plus proprement intuitionniste, l'apport de Hurka mérite une attention particulière, voir par exemple (Hurka 2003). 
telle que, si une certaine chose existe, elle est vraie, tandis que si telle chose n'existe pas, elle est fausse, quelque puisse être la nature du monde » (Moore 1903 : 115). Brentano soutient que les jugements éthiques possèdent une objectivité constitutive qui en garantit la validité et la consistance normative. Il existe toutefois une différence importante de caractère épistémologique : d'après Moore, le concept de «bon» est une simple notion analogue, du point de vue formel, à la notion de jaune; mais tandis que jaune est une notion d'ordre naturel, le bon ne l'est pas. L'erreur la plus répandue en éthique a été le fait de croire qu'il est suffisant de décrire un objet qui possède une qualité déterminée pour connaître la qualité en question ; il s'agit, selon Moore, d'une fausseté du naturalisme qui porte à croire que le bon est une espèce de qualité partielle. Cela conduit Moore à affirmer qu'entre faits et valeurs il existe une dichotomie puisque les propositions descriptives - autour de ce qui est — diffèrent du point de vue de la forme et du contenu des propositions normatives - autour de ce qui doit être. Dans l'introduction aux Principia Ethica (1903), Moore écrit :

Brentano est en accord total avec moi : 1/ en ce qu'il considère toutes les propositions éthiques comme définies par le fait qu'elles prédiquent un seul et unique concept objectif ; 2 /en ce que son classement des propositions aboutit à deux catégories identiques aux miennes; $3 /$ en ce qu'il tient que les propositions du premier genre sont incapables d'être prouvées ; et 4/ eu égard $\mathrm{au}$ genre d'évidence qui est nécessaire et pertinent pour démontrer les propositions du second genre (Moore 1998 : 4-5).

À la lumière de ces constatations, si d'un côté Moore et Brentano partagent l'idée que la bonté intrinsèque est une propriété possédée et prédiquée des objets, de l'autre, selon Moore, cette propriété est aussi fondamentale qu'inanalysable, tandis que pour Brentano elle est analysable dans les termes d'« aimer ce qui est démontré comme juste » (McAlister 1982 : 96). Cela conduit à une certaine circularité épistémique qui apparaît encore plus problématique dans l'économie de la pensée de Brentano, si l'on tient compte du fait que la finalité de ses recherches psychologiques était de classifier et de typologiser les phénomènes psychiques: dans le cas du concept de bien - mais aussi des autres termes éthiques fondamentaux on se trouve en présence d'un phénomène qui possède des qualités spécifiques qui sont autant d'obstacles à sa prise en compte dans la classification des activités psychiques.

Si les affinités et divergences théoriques rappelées jusqu'ici concernent essentiellement l'objet et la modalité par laquelle une objectivation spécifique de la sphère émotive est possible, un aspect du traité éthique de

\section{4}

Bull. anal. phén. XIV 3 (2018)

https://popups.uliege.be/1782-2041/ @ 2018 ULiège BAP 
Brentano que Moore apprécie réside dans le recours implicite au concept d' « intrinsic value », par lequel l'auteur de la Psychologie du point de vue empirique élaborerait une définition $\mathrm{du}$ 《bon en soi» non pas dans les termes d'un concept subjectif mais d'une modalité de référence indirecte, qui conduit à considérer les prédicats de valeur comme «bon » et «juste» en connexion avec les modalités qui se réfèrent à elles. Brentano, surtout dans la phase initiale de sa réflexion philosophique, considérait que les termes «nomment» directement les concepts et indirectement les phénomènes par lesquels les concepts sont acquis ; «bon » désigne un terme qui semble être le concept d'une propriété qui peut être possédée par un objet. Pour expliquer en dernière analyse ce que signifie l'adjectif « bon », on doit donc, en suivant les indications de Brentano, être en état de montrer l'origine du concept que le mot désigne et ceci n'est possible qu'en trouvant le phénomène correspondant. Si l'on tient compte de l'une des thèses fondamentales de la psychologie empirique, c'est-à-dire du fait que les concepts découlent de l'expérience, il reste à savoir si le concept de bien est dérivé de la perception externe ou de la perception interne. La réponse de Brentano est que le concept de bien trouve son origine dans la perception interne. Toutefois, le type de la perception interne qui garantit l'évidence et l'apodicticité du concept de bon est une forme de référence intentionnelle de second niveau qui, en vertu de son contenu spécifique - une manifestation de caractère affectif -, ne peut pas tout à fait se superposer à la perception interne qui garantit l'évidence des phénomènes représentatifs et judicatifs. On pourrait la définir comme une perception interne affective à travers laquelle on n'établit pas tant si l'objet intentionnel est aimé ou haï, mais plutôt s'il est digne d'être aimé ou aimé justement ${ }^{1}$.

La réflexion de Moore sur le concept de valeur intrinsèque essaie de façon plus générale de comprendre si tous les prédicats de valeur sont ou ne sont pas des prédicats subjectifs. Sous cet aspect, la distinction entre valeur objective et valeur intrinsèque est fondamentale :

La vérité, je crois, se tient dans le fait que même si la proposition selon laquelle un type particulier de valeur est intrinsèque, il s'ensuit qu'il doit être

\footnotetext{
${ }^{1}$ À ce propos, McAlister rappelle justement que la notion de «digne d'être aimé » doit être distinguée de la notion de jugement juste ou correct, puisqu'il est nécessaire de distinguer le domaine de la logique de celui de l'éthique: « Si les jugements ont affaire à l'existence ou à la non-existence des objets jugés, les émotions ou les actes de la volonté ont affaire à la valeur de leurs objets indépendamment du fait que ces objets existent, et les deux sphères ne sont donc pas réductibles l'une à l'autre même si elles possèdent des structures parallèles » (McAlister 1983 : 85-86).
} 
objectif, l'implication opposée ne tient en aucune façon, mais au contraire il est parfaitement facile de concevoir des théories par exemple de la bonté, selon laquelle la bonté serait au sens le plus strict objective et pas encore intrinsèque. Il y a donc une différence très importante entre la conception de l'objectivité et celle qui la définit comme intrinsèque (internality); mais en outre, si je ne fais pas erreur, quand les gens parlent d'objectivité de n'importe quel type de valeur, ils confondent presque toujours les deux, à cause du fait que la plupart de ces personnes nient l'être intrinsèque d'une donnée type de valeur, qui affirme sa subjectivité (Moore 1922 : 255).

D'après Moore, le notion de bien est «indéfinissable » car la propriété d' «être bon» ne peut être analysée dans ses caractéristiques constitutives et élémentaires; pour ce motif, elle se présente comme un open question argument. Cette position cognitiviste se fonde sur la thèse selon laquelle les jugements moraux sont des affirmations qui attribuent des propriétés aux choses : la " bonté » n'est pas comme la propriété «rouge » qui peut être perçue ou apprise au moyen des sens. Les propositions éthiques sont, selon Moore, dans la plupart des cas, auto-évidentes. Toutefois, les intuitions morales ne sont pas tant indémontrables parce qu'elles seraient obscures ou probablement fallacieuses, mais en raison même de leurs caractéristiques constitutives. En outre, bien qu'il conçoit les intuitions morales en termes de propositions, il découvre un certain type de validité même dans les «prédispositions »: dans cette perspective, une intuition n'est pas seulement un «état propositionnel », mais une « cognition», non pas un contenu abstrait mais une attitude propositionnelle, probablement une croyance qui possède un contenu déterminé. La distinction entre une acception d'aptitude et une acception propositionnelle du terme intuition est le substrat théorique de la notion de self-evident proposition - les principes éthiques sont naturellement self-evident.

Le renvoi au concept de valeur intrinsèque est d'une importance fondamentale pour comprendre pourquoi l'éthique de Brentano a autant attiré l'attention ces dernières dizaines d'années, en particulier à travers l'interprétation controversée (McAlister 1976 : 151-159) qu'a donnée Chisholm de l'intentionnalité brentanienne. Mais, contrairement à la thèse de Moore selon laquelle Brentano identifierait erronément le concept éthique fondamental de «good in itself» avec celui de «rightly loved» ou de «worthy of love » -, Brentano, comme le relève justement Chisholm ${ }^{1}$, n'affirme pas que

${ }^{1}$ Chisholm se rend compte de cet aspect problématique et il écrit : « La théorie de Brentano se caractérise de façon telle qu'elle peut sembler en conflit avec le caractère objectif que je lui ai attribué. Brentano affirme que les termes "bon", "mauvais" 
la justesse est un concept quantitatif, mais plutôt qu'elle est le résultat d'une activité psychique spécifique, à savoir d'un Vorzugsakt. D'après les indications fournies par Brentano dans l'Ursprung, le fait de dire qu'une chose est préférable à une autre chose signifie en effet, d'un côté, présupposer la compréhension d'une telle relation de manière analogue à la reconnaissance objective d'actes d'amour et de haine déterminés comme justes ou à travers la perception interne, et de l'autre, admettre la possibilité que l'acte de préférence puisse se constituer, dans certains cas, à travers la détermination spécifique des valeurs relatives.

Selon Chisholm, la théorie de Brentano se configure comme une théorie de la valeur intrinsèque de type objectif, parce qu'elle se fonde sur l'idée d'une analogie entre jugements et mouvements affectifs : d'un côté un même jugement ne peut pas être à la fois correct et non correct, de l'autre une même émotion ne peut pas être à la fois correcte et non correcte. Pour être plus précis, il s'agit selon Chisholm (Chisholm $1998: 4$ ) d'une théorie pluraliste qui, contrairement à ce que soutenait Moore (Moore $1998: 5$ ), est en mesure de supporter une vision organique et holistique de la valeur. Afin de résoudre ces difficultés d'un point de vue plus proprement axiologique, Chisholm propose d'avoir recours à ce qu'il appelle une «isolation approach». Une telle opération permettrait de déterminer une forme fondamentale et objective de valeur intrinsèque au moyen d'un critère de choix basé sur l'«intrinsic preferability» - l'innere Vorzüglichkeit de Brentano - et il affirme que cette opération permet non seulement une définition adéquate du concept de valeur intrinsèque, mais encore de réduire l'axiologique au déontologique.

Si l'on suit les thèses de la psychologie descriptive de Brentano, la théorie de la valeur qu'il propose se fonde sur la thèse qu'il existe entre faits et valeurs une différence non seulement épistémologique, comme le soutient Moore, mais également ontologique. Selon Brentano, il n'existe qu'une seule réalité et cette réalité est immanente au sujet, tandis que les modalités de référence intentionnelles sont multiples. Le fait qu'il y a trois classes

et "meilleur" sont syncatégorématiques quand ils sont utilisés en connexion avec le concept de valeur intrinsèque. Par cette affirmation Brentano réfute les théories basées sur la prédication de la valeur comme celles qui sont présupposées par Moore et Scheler. Le terme "bon" possède, dans son intention, une simple qualité comparable à celle qui est possédée par le mot jaune ou bleu. Avec cette affirmation, Brentano exprimerait ce qu'il a défini comme son "réisme". Il est entré dans cette nouvelle phase de pensée en 1905 : après laquelle il n'a plus soutenu l'existence de la classe des "non-choses" en tant qu'objets abstraits, propositions ou état de choses » (cf. Chisholm $1986: 5)$. 
d'activités psychiques ne signifie pas qu'il y a trois types de réalité, car toute émotion est fondée sur la présentation — ou sur le jugement. Si les présentations fournissent aux émotions leurs objets, il s'ensuit qu'il n'y a pas deux objets différents mais seulement deux modalités différentes de concevoir le même objet. Cette dernière idée est d'une importance capitale pour comprendre en quel sens Brentano défend un « réalisme moral».

\section{Bibliographie}

Albertazzi, Liliana (2006). Immanent Realism. An Introduction to Brentano, Dordrecht, Springer.

Audi, Robert (2004). The Good in the Right. A Theory of Intuition and Intrinsic Value, Princeton, Princeton University Press.

Baumgartner, Wilhelm \& Pasquarella, Lynn (2004). «Brentano's value theory: beauty, goodness and the concept of correct emotion ». In. D. Jacquette (ed.), The Cambridge Companion to Brentano. Cambridge, Cambridge University Press, p. $220-236$.

Brentano, Franz (1952). Grundlegung und Aufbau der Ethik, Nach den Vorlesungen über Praktische Philosophie. Franziska Mayer-Hillebrand (Hrsg.), Hamburg, Meiner.

- (1969). Vom Ursprung sittlicher Erkenntnis, Hamburg, Meiner. Trad. par Marc de Launay et Jean-Claude Gens, L'origine de la connaissance morale suivi de La doctrine du jugement correct, Paris, Gallimard, 2003.

- (1973 [1874]). Psychologie vom empirischen Standpunkt. Meiner, Hamburg. Trad. par M. de Gandillac. Nouvelle édition revue et présentée par J-F Courtine, Psychologie du point de vue empirique. Paris, J. Vrin, 2008.

Chisholm, Roderick (1986). Brentano and Intrinsic Value, Cambridge MA, Cambridge University Press.

Danielsson, Sven \& Olson Jonas (2007). « Brentano and Buck-Passers », Mind, vol. $116, \mathrm{~N}^{\circ} 463$, p. $511-522$.

Findlay, John N. (1970). Axiological Ethics, New York, Palgrave.

Fisette, Denis (2011). «Love and Hate: Brentano and Stumpf on Emotions and Sense Feelings». In S. Bonacchi \& G-J. Boudewijnse (eds.), Carl Stumpf From Philosophial Reflection to Interdisciplinary Scientific Investigation, Wien, Krammer, p. 37- 49.

Huemer, Michael (2005). Ethical Intuitionism, New York, Palgrave MacMillan.

Hurka, Thomas (2003). "Moore in the Middle», Ethics, vol. 113, Centenary Symposium on G.E. Moore's Principia, 3, p. 599-628.

Kriegel, Uriah (2017). «Brentano's Evaluative-Attitudinal Account of Will and Emotion », Revue philosophique de la France et de l'étranger, Tome 142, $\mathrm{n}^{\circ} 4$, p. $529-558$. 
Kriegel, Uriah (2018). Brentano Philosophical System. Mind, Being, Value. Oxford, Oxford University Press.

Kraus, Oskar (1937). Die Werttheorien-Geschichte und Kritik, Brünn-Wien-Leipzig, Rudolf M. Rohrer.

— (1972 [1925]), « Einleitung des Herausgebers », in F. Brentano, Psychologie vom empirschen Standpunkt. ZweiterBand. Von der Klassifikation der psychischen Phänomene, Hamburg, Meiner.

Massin, Olivier (2013). «The Intentionality of Pleasures and Feelings, A Brentanian Approach». In D. Fisette \& G. Frechette (éds.), Themes from Brentano, Amsterdam, Rodopi, p. 307-338.

McAlister, Linda (1976). «Chisholm and Brentano on Intentionality ». In L. McAlister (ed.), The Philosophy of Franz Brentano, London, Durckworth, p. 151-179.

- (1982). The Development of Franz Brentano's Ethics, Amsterdam, Rodopi. (Réédité partiellement in M. Antonelli \& F. Boccaccini (eds.), Franz Brentano Critical Assessment, vol. 4, «Ethics, Aesthetics, Religion », London-New York, Routledge, 2018 (sous presse)).

Montague, Michael (2017). «A Contemporary View of Brentano's Theory of Emotion », The Monist, 100, p. 64-87.

Moore, George E. (1903). «Review: The Origin of the Knowledge of Right and Wrong », International Journal of Ethics, vol. 14, 1, (Réédité in M. Antonelli \& F. Boccaccini (eds.), Franz Brentano Critical Assessment (2018). vol. 4, « Ethics, Aesthetics, Religion », London-New York, Routledge, 2018 (sous presse)).

- (1922). Philosophical Studies, Kegan Paul, Trench Trubner \& CO., LTD, New York.

- (1998), Principia Ethica, Paris, Puf.

Olson, Jonas (2017a). «Brentano's metaethics ». In U. Kriegel (ed.), The Routledge Handbook of Brentano and the Brentano School, New York, Routledge, p. 187195.

- (2017b). «Two Kinds of Ethical Intuitionism: Brentano's and Reids », The Monist, 100, p. 106-119.

Rønnow-Rasmussen, Toni. \& Zimmermann Michael J., (eds.) (2005). Recent Works on Intrinsic Value, Dordrecht, Springer.

Seron, Denis (2008). «Sur l'analogie entre théorie et pratique chez Brentano », Bulletin d'Analyse Phénoménologique, IV, 3,p. 23-51.

Stumpf, Carl (1906). «Über Gefühlsempfindungen », Bericht über den II. Kongre $\beta$ für experimentelle Psychologie in Würzburg vom 18-21. April 1906, F. Schumann (Hrsg.), Leipzig, Barth, p. 209-213.

Scheler, Max (2000). Der Formalismus in der Ethik und die materiale Wertethik. Neuer Versuch der Grundlegung eines ethischen Personalismus, in Gesammelte werke, Bd II., Studiensausgabe, 8. Aufl., Nach der 7, Bonn, Bouvier.

Stratton-Lake, Philip (2006). Ethical Intuitionism: Re-evaluations, New York, Oxford. 